\title{
A Multiple Isolated-Input Network With Common Output*
}

\author{
C. M. Allred and C. C. Cook
}

(March 31, 1960)

\begin{abstract}
A circuit is described having $n$ inputs which are isolated from each other but fed into a common output. Theoretically, the circuit presents matched impedances at each of the inputs and the output and has minimum transmission loss. Design equations are presented for the general case and performance data is given for a two-input and a three-input unit.
\end{abstract}

\section{Introduction}

In certain highly accurate and precise attenuation measurements a network having multiple inputs and a common output was needed. The following characteristics were to be met:

(a) The network should be symmetrical; that is, each input should have identical electrical characteristics with respect to the output and to every other input.

(b) The inputs should be isolated from each other; that is, an excitation applied to any input should produce negligible response at any other input.

(c) The network should have maximum sensitivity; that is, a change in the excitation at any given input should produce as large a power change in the output as possible.

(d) The input impedance is specified.

(e) Stability considerations require the use of passive, linear, bilateral elements: resistors, capacitors, and inductors.

(f) The system should be unbalanced so the inputs and output have a common ground.

(g) The network need have these characteristics at a single frequency only.

The design equations for such a network are given for a circuit with " $n$ " input terminals. Experimental results for $n=2$ and $n=3$ are presented.

\section{Theory}

\subsection{General}

A network possessing the above characteristics will, of course, have properties not explicitly stated above. It is shown below that even less restrictive networks (those meeting only requirements (b), (e), and part of (a), where only the ratio of output power to input power for single input excitation need be equal for all inputs) must contain losses. This is true even when ideal lossless capacitors and inductances are available. These losses affect the sensitivity requirement (c), and therefore should be minimized. However, there is a limit to which the losses can be reduced.

Let $\alpha$, called the sensitivity, be defined as:

$$
\alpha=\frac{P_{o k}}{P_{k}}
$$

\footnotetext{
* Contributions from Radio Standards Laboratory, National Bureau of Stand-
} ards, Boulder, Colo. where $P_{o k}$ is the output power when only the $k$ th input is excited. The power into the $k$ th input is $P_{k}$.

It is shown in the following that the maximum attainable $\alpha$ is:

$$
\alpha_{\max }=\frac{1}{n}
$$

Thus a specific network may well have an $\alpha$ which is less than $1 / n$, but no network, no matter how nearly ideal, if it fulfills even the lesser requirements, can have an $\alpha$ greater than $1 / n$.

To obtain the maximum value of $\alpha$, consider a network with $n$ inputs where the input impedances and phase shifts between output and input may be arbitrarily different for each input. However, it is assumed that the actual ratio, $\alpha$, of the output power, $P_{o k}$, to the input power, $P_{k}$, where only the $k$ th input is excited, is the same for each input and there is complete isolation between all inputs. Thus

$$
\alpha=\frac{P_{o k}}{P_{k}}=\frac{E_{o k}^{2}}{E_{k}^{2}} \frac{G_{L}}{G_{k}}
$$

for $k=1,2, \ldots, n$. Here $E_{o k}$ is the magnitude (rms) of the voltage across the load when only the $k$ th input is excited with a voltage of magnitude $E_{k}$.

If all inputs are excited from coherent sources with magnitudes and phases such that the input powers are equal and the output voltages are all in phase, the following relationships hold.

$$
\begin{aligned}
P_{\mathrm{in}} & =n P_{k} \\
P_{o} & =\left(n E_{o k}\right)^{2} G_{L}=n^{2} P_{o k},
\end{aligned}
$$

where $P_{\text {in }}$ and $P_{o}$ are the total input and output powers, respectively.

The conservation of energy requires that

$$
P_{\text {in }}=P_{o}+P_{c}
$$

or

$$
n P_{k}=n^{2} P_{o k}+P_{c},
$$

where $\mathrm{P}_{c}$ is the power lost in the circuitry.

Rearranging terms and using the definition of $\alpha$, one obtains

$$
\alpha=\frac{1}{n}-\frac{P_{c}}{n^{2} P_{k}} .
$$


The maximum value of $\alpha$ occurs when $P_{c}=0$ (passive networks only are considered). Thus

$$
\alpha_{\max }=\frac{1}{n} .
$$

The following aspects of the discussion may well be emphasized:

(a) The maximum possible value of $\alpha, \alpha_{\max }$, is a property of the network arising from the requirement of equal sensitivities for all inputs and total isolation between the inputs.

(b) The quantities, $\alpha$ and $\alpha_{\max }$, are defined only for single input excitation.

(c) The ratio of the total output power to total input power when more than one input is excited is not specified and may vary between the limits of zero and unity depending on the levels and phase relations between the excitations.

(d) The above proof does not imply that a network with maximum $\alpha$ contains no dissipative elements but merely that when all inputs are excited so as to have outputs that are equal in magnitude and phase, then no power must be dissipated within the network. This is a case where the ratio of the total power out to total power in is unity.

\subsection{The Actual Circuit}

While there are other networks possessing the desired characteristics, the one used here is a modification of the familiar bridged- $T$ infinite rejection network. ${ }^{1}$

The familiar bridged- $T$ network is shown in figure 1. This may be redrawn in the form of figure 2. The inductance $L^{\prime}$, which does not alter the infinite rejection characteristics, is added to make the input admittance purely conductive. Here, $R$ is considered the load, $R_{L}$, which would be applied to the output terminals and is not included in the drawing. The circuit extended to four inputs is shown in figure 3 .

The extension to $n$ inputs is apparent. This consists of coupling each input with the output through a capacitor, $C$, and each input with every

1 G. E. Valley and H. Wallman, Vacuum tube amplifiers, ch. 10, Radiation Lab. Ser. (McGraw Hill Book Co., Inc., New York, N.Y., 1948).

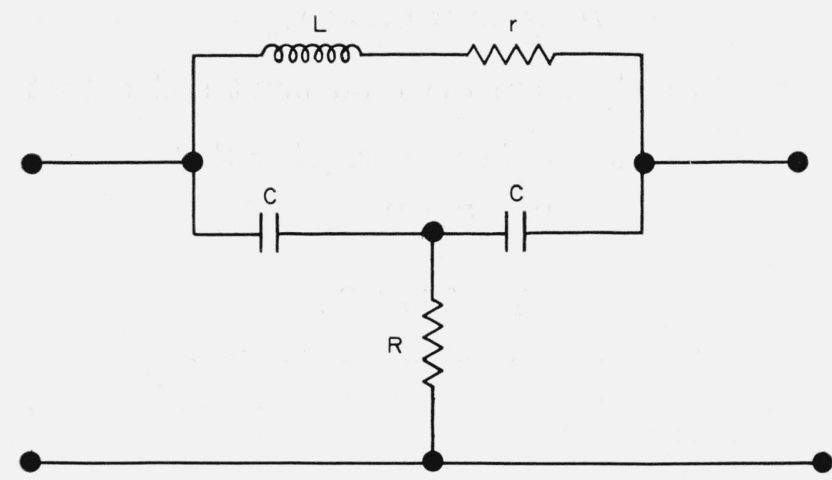

FIGURE 1. Bridged-T infinite rejection network.

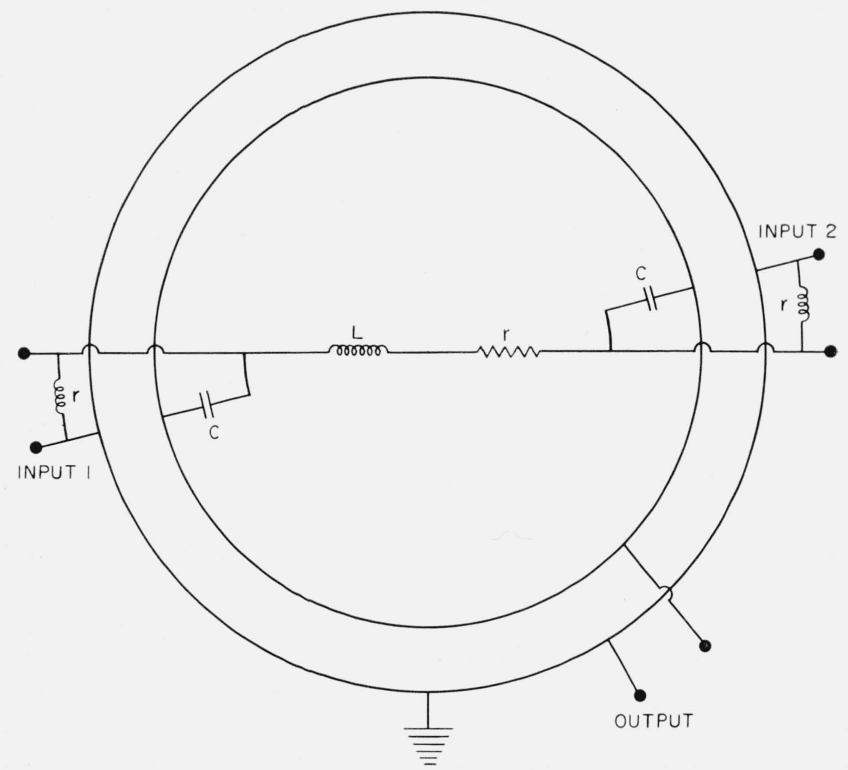

FIGURE 2. Bridged-T network in different form.

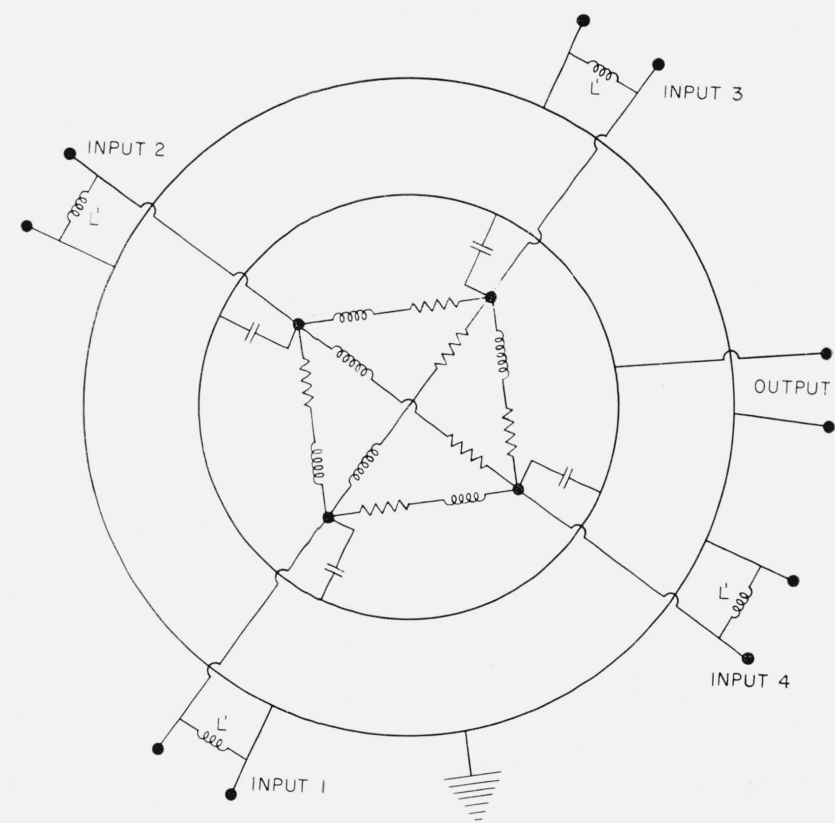

FiguRE 3. Four-input infinite rejection network

other input through a resistance-inductance series circuit $(r$ and $L)$. Again, a shunt inductance, $L^{\prime}$, is placed across each input in order to make the input admittance purely conductive. The nodal equations ${ }^{2}$ may be readily written for the $n$ input case. They are:

${ }^{2}$ H. W. Bode, Network analysis and feedback amplifier design, ch. 1 (D. Van Nostrand Co., Inc., 250 Fourth Avenue, New York, N.Y., 1945); M. E. Van Valkenburg, Network analysis, ch. 3 (Prentice Hall, Inc., New York, N.Y. $1955)$. 


$$
\begin{aligned}
& e_{1}\left(j \omega c+\frac{n-1}{r+j \omega L}+\frac{1}{j \omega L^{\prime}}\right) \\
& \quad-\left(e_{2}+e_{3}+\ldots+e_{n}\right) \frac{1}{r+j \omega L}-e_{0} j \omega c=i_{1}
\end{aligned}
$$

$$
\begin{aligned}
& e_{2}\left(j \omega c+\frac{n-1}{r+j \omega L}+\frac{1}{j \omega L^{\prime}}\right) \\
& -\left(e_{1}+e_{3}+\ldots+e_{n}\right) \frac{1}{r+j \omega L}-e_{0} j \omega c=i_{2}
\end{aligned}
$$

$$
\begin{aligned}
e_{n}(j \omega c & \left.+\frac{n-1}{r+j \omega L}+\frac{1}{j \omega L^{\prime}}\right) \\
& -\left(e_{1}+e_{2}+\ldots+e_{n-1}\right) \frac{1}{r+j \omega L}-e_{0} j \omega c=i_{n} \\
- & \left(e_{1}+e_{2}+\ldots+e_{n}\right) j \omega c+e_{0}\left(n j \omega c+\frac{1}{R_{L}}\right)=0 .
\end{aligned}
$$

If all applied currents are zero except one, say $i_{1}$, and all input voltages are required to be zero except $e_{1}$, the equations reduce to:

$$
\begin{gathered}
e_{1}\left(j \omega c+\frac{n-1}{r+j \omega L}+\frac{1}{j \omega L^{\prime}}\right)-e_{0} j \omega c=i_{1} \\
-e_{1} \frac{1}{r+j \omega L}-e_{0} j \omega c=0 \\
-e_{1} j \omega c+e_{0}\left(n j \omega c+\frac{1}{R_{L}}\right)=0 .
\end{gathered}
$$

Equations (6) and (7) require relationships between the components which are:

$$
\begin{aligned}
\omega^{2} c^{2} r R_{L} & =1 \\
\omega L \omega c & =n .
\end{aligned}
$$

Subtracting eq (6) from eq (5) and obtaining the ratio, $e_{1} / i_{1}$, the input impedance is found. If this is required to be $R_{0}$ (purely resistive), the following relationship holds:

$$
\frac{1}{R_{0}}=j \omega c+\frac{n}{r+j \omega L}+\frac{1}{j \omega L^{\prime}} .
$$

On equating real and imaginary components:

$$
\begin{gathered}
R_{0}=\frac{r^{2}+\omega^{2} L^{2}}{r n} \\
\omega c-\frac{1}{\omega L^{\prime}}-\frac{n \omega L}{r^{2}+\omega^{2} L^{2}}=0 .
\end{gathered}
$$

From these equations, the various relationships between the circuit elements necessary to obtain the desired network characteristics may be obtained.

\section{The Design Equations}

The relationships between the various components that produce the desired response described above are given below. These are presented in terms of the parameters $Q \quad(Q=\omega L / r)$, the specified input resistance, $R_{0}$, the number of inputs, $n$, and the angular frequency, $\omega$.

$$
\begin{aligned}
\frac{P_{o k}}{P_{k}} & =\frac{1}{n}(\text { for excitation at one input only) } \\
\frac{E_{o k}}{E_{k}} & =\frac{Q}{n \sqrt{1+Q^{2}}} e^{j \theta} \\
\tan \theta & =\frac{1}{Q} \equiv \frac{r}{\omega L} \\
R_{L} & =\frac{R_{0}}{n} \frac{Q^{2}}{1+Q^{2}} \\
r & =\frac{n R_{0}}{1+Q^{2}} \\
c & =\frac{1}{R_{0} \omega} \frac{1+Q^{2}}{Q} \\
L & =\frac{n R_{0}}{\omega} \frac{Q}{1+Q^{2}} \\
Z_{\text {in }} & =R_{0}+j 0 \\
Z_{\text {out }} & =\frac{R_{0}}{n} \frac{Q^{2}}{1+Q^{2}}=R_{L} \\
L^{\prime} & =\frac{R_{0} Q}{\omega}
\end{aligned}
$$

where $E_{o k}$ is the output voltage across $R_{L}$ when $E_{k}$ is applied across the $k$ th input (the only input excited); and $Z_{\text {out }}$ is the output impedance of the network, i.e., the impedance seen by $R_{L}$ looking back into the network.

\section{Experimental Results}

Two units to operate at a frequency of $30 \mathrm{Mc}$ were constructed. One unit (fig. 4) had two inputs and the other (fig. 5) three inputs.

Stray capacitance, lead inductance, and intercoupling between elements make it necessary to change the component values somewhat from the calculated values. Also, by letting the load impedance be complex, another parameter is available in the design. Since in the application intended for the units, the load impedance was arbitrary, this property was used. Thus the load is not purely resistive.

\section{Two-Input Unit}

Isolation between inputs $110 \mathrm{db}$ Input impedance:

Input 1 $50.4+j 0$ ohms

Input 2 $50.9+j 0 \mathrm{ohms}$ 
Sensitivity, $\alpha$ :

Input 1 to output

$4 \mathrm{db}$

Input 2 to output

$4 \mathrm{db}$

Load impedance

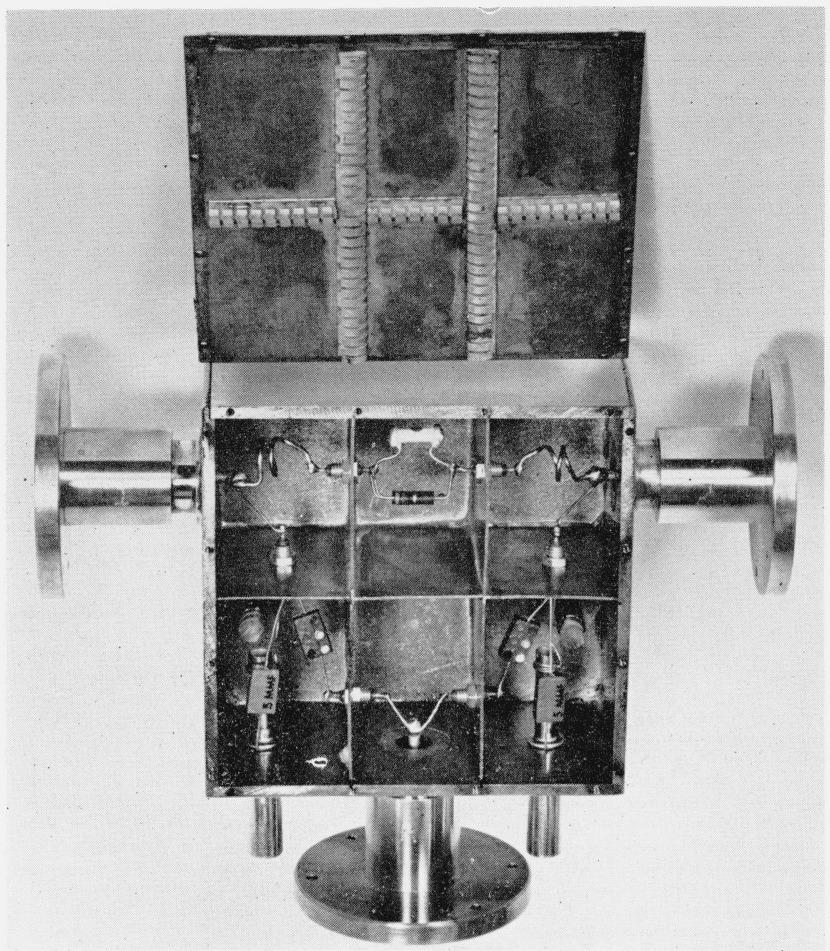

Figure 4. Two-input unit.
Three-Input Unit

Isolation between inputs:

Input 1 and input $2 \ldots \ldots . .57 .5 \mathrm{db}$

Input 1 and input $3 \ldots \ldots \ldots 77.5 \mathrm{db}$

Input 2 and input $3 \ldots \ldots$ _.... $76.0 \mathrm{db}$

Input impedance:

Input $1 \ldots \ldots+\ldots .30 \mathrm{ohms}$

Input $2 \ldots \ldots \ldots 50.0+j 0 \mathrm{ohms}$

Input $3 \ldots \ldots \ldots$ _......... $50.1+j 0 \mathrm{ohms}$

Sensitivity, $\alpha$ :

Input 1 to output _....... $6 \mathrm{db}$

Input 2 to output _...... $6 \mathrm{db}$

Input 3 to output _....... $6 \mathrm{db}$

Load impedance

\section{Conclusions}

A useful network for unique applications in the field of precision measurements was obtained. It may be of interest to note that the properties of the network depend strongly on the load impedance except when a sufficient number of inputs is excited in a manner to produce a null at the load. For this case there can be no dependence on the load impedance as the load currents and voltages are zero. Also for this case, the total input power is dissipated entirely within the network.

The authors express their appreciation to Willard H. Long for the mechanical construction and William W. Kingston for his help in wiring, adjusting, and testing the units.

(Paper 64C3-40)
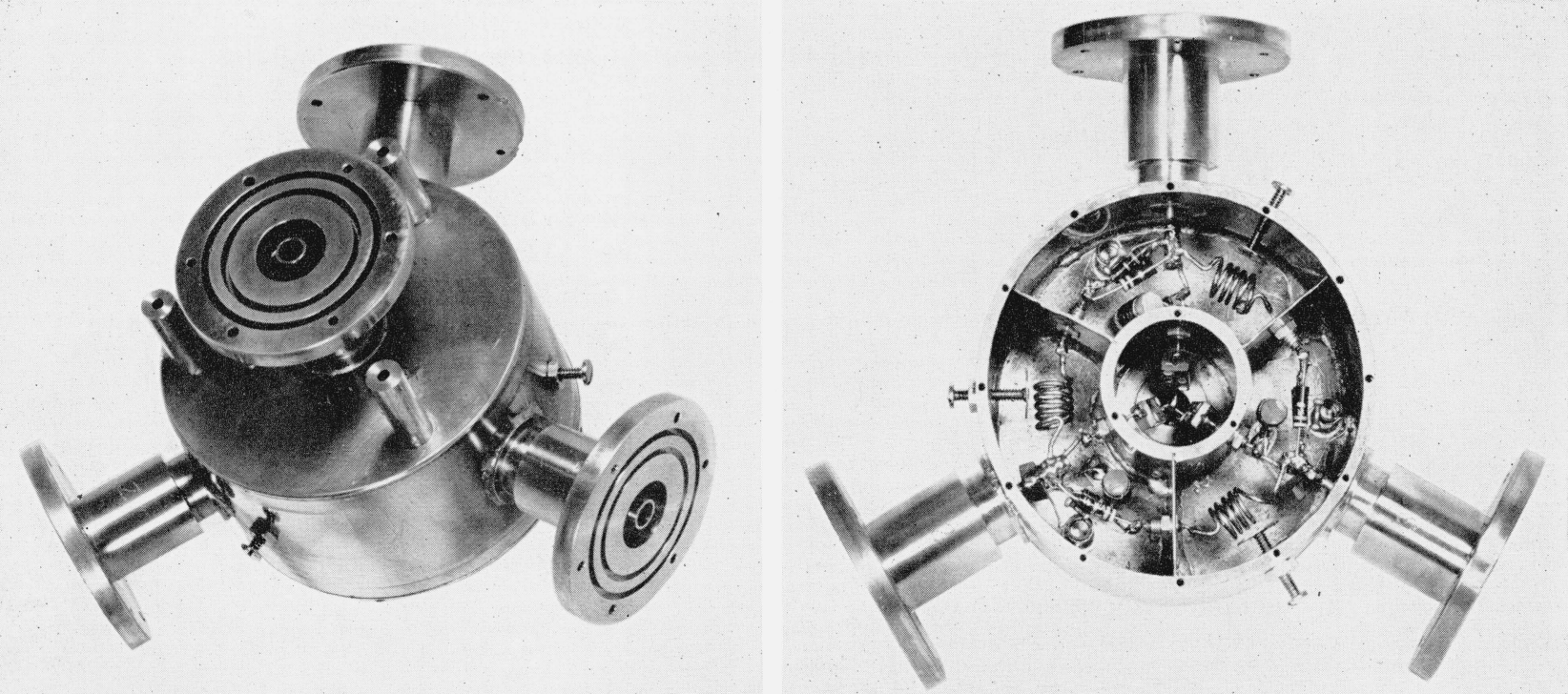

Figure 5. Three-input unit. 